\title{
The Sub-Parsec-Scale Structure and Evolution of Centaurus A at 8.4 and $22.2 \mathrm{GHz}$
}

\section{S. J. Tingay}

Jet Propulsion Laboratory, Caltech, Pasadena, CA 91109, U.S.A.

D. L. Jauncey, J. E. Reynolds, \& A. K. Tzioumis

CSIRO, Australia Telescope National Facility, NSW 2121, Australia

R. A. Preston, D. L. Jones, D. W. Murphy, \& D. L. Meier

Jet Propulsion Laboratory, Caltech, Pasadena, CA 91109, U.S.A.

J. E. J. Lovell, P. M. McCulloch, \& M. E. Costa

University of Tasmania, Hobart, Tasmania 7001, Australia

G. Nicolson \& J. F. H. Quick

Hartebeesthoek Radio Astronomy Observatory, Krugersdorp, South Africa

Abstract. We have been observing the sub-parsec-scale radio jet in the nucleus of Centaurus $\mathrm{A}$, the closest active radio galaxy, at $8.4 \mathrm{GHz}$ over the last 6 years with the SHEVE array and most recently with the VLBA at 8.4 and $22.2 \mathrm{GHz}$. In this paper we will review the results of these observations and give a brief summary of our interpretation, concentrating on the evolution of the sub-parsec-scale jet structure with time; subluminal component motions and rapid short timescale evolution. A full description of this work is soon to be published in a major journal article.

\section{Centaurus A-Results to Date}

Centaurus A has always been a high priority target for the Southern Hemisphere VLBI Experiment (SHEVE; Preston et al. 1989, Jauncey et al. 1994) array due to its proximity - it is the closest active radio galaxy to us, at a distance of approximately 3.5 Mpc (Hui et al. 1993).

The first high resolution interferometry results for Centaurus $A$ were reported by Wade et al. (1971). With the later SHEVE array, Meier et al. (1989) determined a model for the source from multifrequency observations, and Meier et al. (1993) and Tingay et al. (1994) reported on the detailed source structure at $8.4 \mathrm{GHz}$. On the milliarcsecond-scale Centaurus A consists of a bright, compact nucleus and a bright jet which extends toward the north-east. As the number of SHEVE observations at $8.4 \mathrm{GHz}$ grew, and observations were made at $4.8 \mathrm{GHz}$, it became clear that the source structure is strongly frequency and time dependent (Jauncey et al. 1995, Tingay et al. 1996, Preston et al. 1996). In addition, by using the SHEVE array simultaneously with the VLBA, we have been able to produce the most robust VLBI datasets yet for Centaurus A, revealing the presence of a counterjet on sub-parsec-scales (Jones et al. 1996).

Most recently, we have been utilizing the VLBA alone to observe Centaurus $\mathrm{A}$ at $8.4 \mathrm{GHz}$ and $22.2 \mathrm{GHz}$. The VLBA is ideal for obtaining repeatable $(u, v)$ coverage with short periods between observing epochs. For Centaurus A this capability is vital, since the source structure has been seen to vary strongly at $8.4 \mathrm{GHz}$ over periods less than 3 months in duration. At the higher observing frequency of $22.2 \mathrm{GHz}$ the VLBA is also extremely useful since the linear resolution routinely achieved is as high as $0.02 \mathrm{pc}$ at the source. 


\section{Discussion}

Several components are regularly detected in the parsec-scale jet of Centaurus A which, over long periods of time, appear to be traveling away from the core with a significantly subluminal apparent motion $\sim 0.1 \mathrm{c}$. However, on much shorter timescales of a few months, several episodes of apparent rapid evolution internal to the components have been observed, implying apparent speeds of $>0.45 \mathrm{c}$, much greater than the average long term motion, perhaps indicating that the long-term $0.1 \mathrm{c}$ motion is a pattern speed on a much faster underlying flow. This behavior appears to bear a remarkable similarity to what has already been seen on the parsec-scale in M87 (e.g., Biretta 1996). The $\sim 0.1 \mathrm{c}$ average component speeds are not consistent with constraints from observations of the counterjet, but the $>0.45 \mathrm{c}$ inferred speeds are, placing the jet axis at $50^{\circ}-70^{\circ}$ to our line of sight - agreeing qualitatively with the large-scale radio morphology of Centaurus A (see Preston et al., these Proceedings, p. 91).

Acknowledgments. The Australia Telescope is operated as a national facility by the CSIRO. Part of this work has been undertaken at the Jet Propulsion Laboratory, California Institute of Technology under contract to the National Aeronautics and Space Administration. Part of this research was conducted while S.J.T. held an NRC-JPL/NASA Research Associateship. The National Radio Astronomy Observatory is a facility of the National Science Foundation, operated under a cooperative agreement by Associated Universities, Inc.

\section{References}

Biretta, J. A. 1996. in Energy Transport in Radio Galaxies and Quasars, eds. P. E. Hardee, A. H. Bridle, \& J. A. Zensus, (San Francisco: ASP), 187-198.

Jauncey, D. L., et al. 1995. Proc.Nat.Acad.Sci.USA, 92, 11368-11370.

Jauncey, D. L., et al. 1994. in IAU Symp. 158, Very High Resolution Imaging, eds. J. G. Robertson, \& W. J. Tango (Dordrecht: Kluwer), 131-133.

Jones, D. L., et al. 1996. $A p J, 466, \mathrm{~L} 63-65$.

Meier, D.L., et al. 1993. in Sub-arcsecond Radio Astronomy, eds. R. J. Davis, \& R. S. Booth (Cambridge: Cambridge University Press), 201-203.

Meier, D. L., et al. 1989. $A J, 98,27-35$.

Preston, R. A., et al. 1996. in IAU Symp. 175, Extragalactic Radio Sources, eds. R. Ekers, C. Fanti, \& L. Padrielli (Dordrecht: Kluwer), 21-22.

Preston, R. A., et al. 1989. $A J$, 98, 1-26.

Preston, R. A., et al. 1983. ApJ, 266, L93-95.

Tingay, S. J., et al. 1996. in Energy Transport in Radio Galaxies and Quasars, eds. P. E. Hardee, A. H. Bridle, \& J. A. Zensus (San Francisco: Astronomical Society of the Pacific), 215-219.

Tingay, S. J., et al. 1994. Aust. J. Phys., 47, 619-624. 